PROOF

Copyrighted Material

For Review Only • Not for Distribution

Page Copy

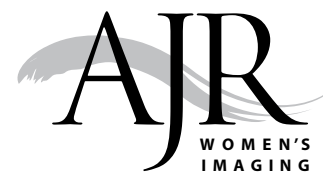

Kavous Firouznia'

Hossein Ghanaati ${ }^{1}$

Mina Sanaati2

Amir H. Jalali ${ }^{3}$

Madjid Shakiba ${ }^{3}$

Firouznia K, Ghanaati H, Sanaati M, Jalali AH, Shakiba M

Keywords: fibroid, pregnancy, therapeutic embolization, uterine artery

\section{DOI:10.2214/AJR.07.3904}

Received February 27, 2008; accepted after revision November 12, 2008.

Please see the commentary on this article, which appears on the following pages.

'Department of Radiology, Medical Imaging Center, Tehran University of Medical Sciences, University of Tehran, Keshavarz Blvd., Tehran 1419733141, Iran. Address correspondence to K. Firouznia.

${ }^{2}$ Department of Gynecology, Eghbal Hospital, Tehran, Iran.

${ }^{3}$ Research Unit, Medical Imaging Center, Tehran University of Medical Sciences, University of Tehran, Tehran, Iran.

AJR2009; 192:1-5

0361-803X/09/1926-1

(c) American Roentgen Ray Society

\title{
Pregnancy After Uterine Artery Embolization for Symptomatic Fibroids: A Series of I5 Pregnancies
}

OBJECTIVE. The purpose of this study was to report on pregnancies and their outcome after uterine artery embolization for uterine fibroids.

SUBJECTS AND METHODS. From 2001 to 2004, 102 patients (mean age, [ \pm SD] $35.7 \pm 6.4$ years; range, $20-48$ years) with symptomatic uterine fibroids underwent uterine artery embolization with 500 - to $710-\mu \mathrm{m}$ polyvinyl alcohol particles. The mean uterine volume was $552 \pm 649 \mathrm{~cm}^{3}$ (range, 94-4,656 $\mathrm{cm}^{3}$ ), and the dominant fibroid size was $228 \pm 359 \mathrm{~cm}^{3}$ (range, 14-2,618 $\mathrm{cm}^{3}$ ) before the procedure. During the 2-year follow-up period, the patients were asked whether they were trying to achieve pregnancy and whether they were successful. We obtained pregnancy and obstetric records of the pregnant women from physicians' offices and hospitals.

RESULTS. Among 102 women who underwent bilateral uterine artery embolization, 23 $(22.5 \%)$ were seeking to become pregnant, and 14 of the $23(61 \%)$ became pregnant, nine having been nulliparous. One patient had two pregnancies. Fourteen pregnancies were spontaneous, and one was achieved by zygote intrafallopian transfer. Two miscarriages occurred, one in the 12th and one in the 16th week of gestation. The other 13 pregnancies went to term, were uncomplicated, and ended in elective cesarean delivery. All of the neonates were healthy with Apgar scores greater than 8. The mean weight of the neonates was 3,274 $\pm 514.4 \mathrm{~g}$ (range, 2,100-3,950 g). One neonate was small for gestational age (2,100 g).

CONCLUSION. Uterine artery embolization can serve as a substitute for invasive operations such as hysterectomy and myomectomy. Additional studies, including prospective, randomized comparisons with myomectomy, should be performed to ascertain whether uterine artery embolization is a safe procedure for women who want to preserve their fertility.

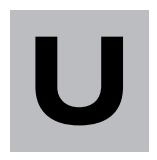
terine fibroids are among the most common benign tumors of the female pelvis that cause abnormal menstrual bleeding. Uterine artery embolization (UAE) in the management of uterine myomas was first reported by Ravina et al. in 1995 [1]. Their promising results were confirmed in several uncontrolled cohort studies, which showed an approximately $50 \%$ decrease in uterine volume and relief of symptoms in $80-90 \%$ of patients [2]. The advantages of UAE include lower cost and more rapid recovery than can be achieved with conventional therapies [35]. The efficacy of uterine embolization in the management of pelvic hemorrhage has been well documented in obstetrics and gynecology [6-8]. Successful pregnancy outcome after embolization for obstetric hemorrhage $[9,10]$, gestational trophoblastic tumor [11], arteriovenous anomaly of the uterus
$[12,13]$, and cervical ectopic pregnancy [1416] has been reported.

The effects of embolization on ovarian function are important when preservation of fertility is desired. Few articles have focused on the effect of UAE for fibroids and its outcome on future fertility and pregnancy. To the best of our knowledge, pregnancy after UAE has been described in the literature in the form of case reports [17-20], a review article [21], and retrospective reports of case series [2229]. As of March 2008, more than 170 patients worldwide had become pregnant after UAE. At a university-affiliated referral hospital in Iran, we evaluated the pregnancies of women who underwent UAE for symptomatic fibroids and desired to become pregnant.

\section{Subjects and Methods}

From November 2001 to November 2004, we performed UAE on 102 women (mean age, $35.7 \pm$ 
6.4 [SD] years; range, 20-48 years) with symptomatic uterine fibroids. The study was approved by our institutional review board, and informed consent was obtained from each patient before the study. Ultrasound and MRI examinations were performed to confirm the diagnosis of fibroids; to determine the size, position, and enhancement of the tumors; and to identify the presence of adenomyosis and other concurrent diseases. The mean uterine volume was $552 \pm 649 \mathrm{~cm}^{3}$ (range, $94-4,656 \mathrm{~cm}^{3}$ ), and the mean dominant fibroid size was $228 \pm 359 \mathrm{~cm}^{3}$ (range, $14-2,618 \mathrm{~cm}^{3}$ ) before the procedure. Women desiring pregnancy were informed of the uncertain effect of embolization on fertility and pregnancy. At least a 6-month interval after embolization and before conception has been recommended [29] depending on fibroid shrinkage and uterine wound healing. We advised patients who wanted to conceive after embolization to wait 1 year.

The settings of the angiographic system (GE DEX DSA, GE Healthcare) were $1,200 \mathrm{~mA}$ and $140 \mathrm{kVp}$. All patients underwent catheterization through a right femoral approach with a 4- or 5-French cobra-shaped catheter without microcatheters. Aortography was performed with a pigtail catheter before pelvic arteriography. The catheter was placed in the abdominal aorta at the level of the renal arteries, and selective catheterization of the uterine arteries was performed as follows.

The tip of a 4- or 5-French cobra-shaped catheter was positioned beyond the junction of the descending and horizontal portions of each uterine artery. Embolization was performed with 500- to 710- $\mu \mathrm{m}$ polyvinyl alcohol particles (Contour, Boston Scientific) injected manually under fluoroscopic control. The particles were slowly injected through the catheter. To avoid retrograde reflux of particles and infiltration to other internal iliac artery side branches, the injection was stopped when arterial flow ceased. When an anastomosis was encountered between the uterine and ovarian arteries, the catheter tip was placed in a position distal to the anastomosis. In some cases, we used gelatin foam particles to occlude the anastomosis temporarily and then performed UAE. We made the gelatin foam particles from sponge sheets (Gelitaspon, Gelita Medical) by cutting the sheet into small fragments with scissors. Postembolization angiography was performed for evaluation of redistribution.

Patients participated in follow-up conducted with questionnaires, telephone interviews, and sonography (EUB-525 system, Hitachi) 1, 3, 6, 12, and 24 months after UAE. At follow-up interviews, patients were asked whether they had been trying to achieve pregnancy and whether they had been successful. We obtained pregnancy and obstetric records of pregnant women from physician's offices and hospitals. Neonatal outcome measurements included gestational age (preterm < 37 weeks), sex, birthweight, and Apgar score. Low birthweight was defined as less than $2,500 \mathrm{~g}$, and very low birthweight was defined as less than $1,500 \mathrm{~g}$.

\section{Results}

Among 102 women who underwent bilateral UAE at our center, $23(22.5 \%)$ intended to become pregnant, and 14 of the 23 (61\%) became pregnant (Table 1). In one patient, conception occurred twice within 12 months (pregnancies 6 and 7). One woman had an unintentional pregnancy.

The mean age at embolization was $31.2 \pm$ 5.5 years (range, 23-40 years). Nine patients $(64 \%)$ had been nulliparous. Four patients $(28.5 \%)$ had a history of spontaneous abortion. Three patients $(21 \%)$ had undergone myomectomy. The mean uterine volume before UAE in the patients who became pregnant was $536 \pm 334 \mathrm{~cm}^{3}$ (range, 161-1,087 $\mathrm{cm}^{3}$ ), and the mean fibroid volume was $228 \pm 252$ $\mathrm{cm}^{3}$ (range, $23.1-723 \mathrm{~cm}^{3}$ ). The mean reduction in uterine volume after 1 year was approximately $37.8 \% \pm 24.6 \%$, and the mean reduction in fibroid volume after 1 year was $64.1 \% \pm 43.1 \%$ in the patients who became pregnant. One patient had a history of infertility with fibroid as the main cause.

Thirteen pregnancies were spontaneous; pregnancy 5 was the result of successful zygote intrafallopian transfer. The mean age at conception was $33.8 \pm 5.2$ years (range, 27 41 years. The mean time from embolization to conception was $30.5 \pm 14.5$ months (range, 2-54 months). There were 13 elective cesarean deliveries; two miscarriages (13\%) occurred, one at 12 weeks and the other at 16

TABLE I: Characteristics of Women Who Became Pregnant After Uterine Artery Embolization

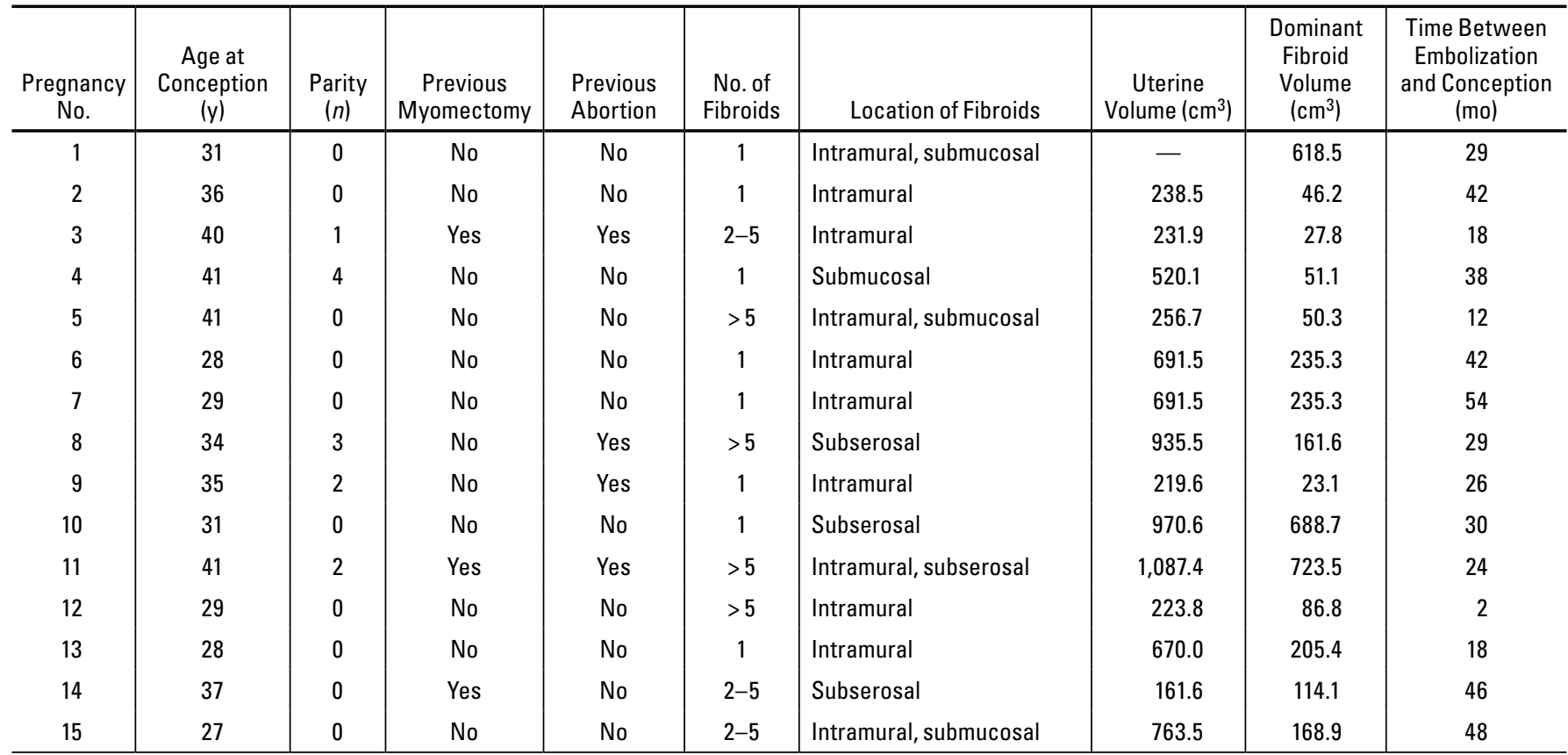

Note-Dash [-] indicates not available. 


\section{Pregnancy After Uterine Artery Embolization}

weeks of gestation. No cause of miscarriage was found in either case. Both patients were 41 years old at the time of conception. The mean age of patients at delivery was $34.7 \pm 5$ years (range, 28-42 years).

One case of postpartum hemorrhage was caused by retained placental tissue. All neonates were born at term and were healthy, with Apgar scores higher than 8. The mean weight of the neonates was $3,274 \pm 514.4 \mathrm{~g}$ (range, 2,100-3,950 g). One neonate was small for gestational age $(2,100 \mathrm{~g})$. The pregnancy and neonatal outcomes are summarized in Table 2. None of the neonates was below the third percentile for weight.

\section{Discussion}

UAE has been found effective in the management of symptomatic uterine fibroids. Spies et al. [30] reported $90 \%$ resolution of heavy bleeding (95\% CI, 86-95\%) and $91 \%$ improvement in bulk-related symptoms (95\% CI, 86-95\%) at 1 year. In an earlier study [31], we found resolution of menstrual bleeding in $81.8 \%$ of the patients and relief of bulk-related symptoms in $84.6 \%$.

The gonads are one of the organs most sensitive to radiation and are in the direct path of the beam during embolization. Nonetheless, the radiation exposure appears to be comparable with that of routine diagnostic imaging procedures [32]. In one study [33], 10 pregnancies occurred in 25 patients after embolization therapy for postpartum hemorrhage. Those authors concluded that women who undergo arterial embolization for obstetric hemorrhage can preserve their fertility and have successful uneventful pregnancies. McLucas et al. [23] evaluated 400 women who underwent UAE from 1996 to 1999. They found that among 139 patients who stated a desire for fertility after UAE, 14 women had 17 pregnancies.

Advanced maternal age adversely affects ovarian function, decreasing the number of good-quality oocytes and resulting in chromosomally abnormal conceptions that rarely develop further. Transient or permanent amenorrhea and other symptoms of ovarian failure have been found in more than $5 \%$ of women who have undergone UAE, especially women older than 45 years [34-36]. However, there have been reports of ovarian failure in younger women $[35,37]$. Ovarian ischemia and loss of ovarian follicles due to nontarget embolization of ovaries are the most likely causes [38, 39]. Spies et al. [34] used serial basal follicle-stimulating hormone assays to evaluate change in ovarian function in 63 patients who had undergone UAE. Those investigators concluded that most of their patients had no change in ovarian function after UAE and that patients 45 years old or older have an approximately $15 \%$ chance of an increase in basal follicle-stimulating hormone level into the perimenopausal range. Results of a prospective study [40] in which the subjects were 20 regularly cycling women indicated no change in ovarian reserve as assessed with basal follicle-stimulating hormone and estradiol levels and ultrasound-based ovarian volume and antral follicle count as long as 12 months after UAE.

In our study, overall pregnancy complications were within the normal ranges of the general obstetric population. All infants in our study were born alive, and none was born preterm. One infant (pregnancy 12) had a low birthweight, and we did not find the exact cause of the low birthweight. The time between embolization and conception, 2 months in this case, may have been too short. This amount of time is not enough for complete involution of fibroids or for development of a collateral arterial supply. Patients who wanted to conceive after embolization were advised to wait 1 year, but this patient did not follow our advice. The optimal time to achieve pregnancy after embolization is unclear. A waiting period of 6 months after UAE and before conception, depending on fibroid shrinkage and the progress of uterine wound healing, has been recommended [29].

Fibroids shrink slowly in most cases; most reductions occur within 6 months to 1 year after UAE. The extent and duration of uterine healing associated with these changes are unknown [23]. Reduction in uterine blood flow caused by bilateral UAE usually is reversed by collateral arterial circulation. In previous studies $[41,42]$ ultrasound imaging and MRI have shown revascularization of the normal myometrium 3-6 months after UAE.

Goldberg et al. [25] compared data on 53 pregnancies after uterine embolization and 139 pregnancies after laparoscopic myomectomy. They found that women who underwent UAE were older and higher in parity and had larger fibroids than women treated with myomectomy $(p<0.001)$. They concluded that women who underwent UAE were at higher risk of spontaneous abortion, postpartum hemorrhage, caesarean delivery, and giving birth to small-for-gestational age infants.

The Royal College of Obstetrics and Gynecology cites a $10-15 \%$ risk of spontaneous miscarriage in the general population, and rates of miscarriage increase twofold to threefold after the age of 40 years [43]. In the Ontario UAE trial, which is the most comprehensive report of pregnancy after UAE with polyvinyl alcohol particles (among 555 women, 22 conceived; three twice, for a total of 25 pregnancies), miscarriages occurred in four women $(16.7 \%)$ [24]. The rate of miscarriage in our patients was $13 \%$ (two cases), and

TABLE 2: Neonatal Outcome After Uterine Artery Embolization

\begin{tabular}{c|c|c}
\hline Pregnancy No. & Type of Delivery & Birth Weight $(\mathrm{kg})$ \\
\hline 1 & Cesarean & 3.510 \\
3 & Cesarean & 3.250 \\
4 & Cesarean & 3.550 \\
5 & Miscarriage & Miscarriage \\
6 & Cesarean & 3.800 \\
7 & Cesarean & 3.000 \\
8 & Cesarean & 2.810 \\
9 & Cesarean & 3.600 \\
10 & Cesarean & 3.300 \\
11 & Cesarean & - \\
12 & Miscarriage & Miscarriage \\
13 & Cesarean & 2.100 \\
14 & Cesarean & - \\
15 & Cesarean & 3.950 \\
\hline
\end{tabular}

Note-Dash [-] indicates definitive weights of these two neonates are not available and were reported by the obstetrician as normal weight. 


\section{Firouznia et al.}

both patients were 41 years old. The spontaneous abortion rate, although based on a small sample population, does not seem to be greater than the rate in the general population.

Residual fibroids after UAE can distort the shape of the uterine cavity and cause malimplantation. This complication can increase the rate of preterm birth and malpresentation, both of which are associated with UAE. The effects of embolization on the stretching ability of the myometrium and mechanisms that initiate labor also can have considerable influence on preterm labor.

In all cases in this study, elective cesarean delivery was performed because of the obstetricians' limited information on pregnancy after UAE. The result was a conservative approach to the management of labor for these patients. Goldberg et al. [25] reported a $63 \%$ rate of cesarean delivery in the UAE group and a rate of $59 \%$ in the laparoscopic myomectomy group. Walker and McDowell [26] reported 56 completed pregnancies in approximately 1,200 UAE patients; 33 pregnancies had successful outcomes, six infants were premature, and 17 pregnancies ended in miscarriage. Among the 33 deliveries, 24 (72.7\%) were cesarean. The authors concluded that compared with the general obstetric population, women who undergo UAE have a significantly greater rate of cesarean delivery.

Advanced maternal age, multiparity, smoking, and previous cesarean delivery are known risks of abnormal placentation [24]. We do not have information about the placentas in our patients. Prenatal ultrasound examinations, however, did not show placental abnormalities. The literature is insufficient for gaining an understanding of the effects of UAE on placental function and fetal growth.

We conclude that UAE can serve as a substitute for invasive operations such as hysterectomy and myomectomy. Additional studies, including prospective randomized comparisons with myomectomy, should be performed to further test the safety and efficacy of UAE in the treatment of women with fibroids who want to preserve their fertility.

\section{References}

1. Ravina JH, Herbreteau D, Cirau-Vigneron N, et al. Arterial embolisation to treat uterine myomata. Lancet 1995; 346:671-672

2. Helmberger TK, Jakobs TF, Reiser MF. Embolization of uterine fibroids. Abdom Imaging 2004; 29:267-277

3. Al-Fozan H, Dufort J, Kaplow M, et al. Cost analysis of myomectomy, hysterectomy and uterine artery embolization. Am J Obstet Gynecol 2002; 187:1401-1404

4. Baker CM, Winkel CA, Subramanian S, et al. Estimated costs for uterine artery embolization and abdominal myomectomy for uterine leiomyomata: a comparative study at a single institution. J Vasc Interv Radiol 2002; 13:1207-1210

5. Smith WJ, Upton E, Shuster EJ, et al. Patient satisfaction and disease specific quality of life after uterine artery embolization. Am J Obstet Gynecol 2004; 190:1697-1703

6. Stancato-Pasik A, Mitty HA, Richard HM, et al. Obstetric embolotherapy: effect on menses and pregnancy. Radiology 1997; 204:791-793

7. Vedantham S, Goodwin S, McLucas B, et al. Uterine artery embolization: an underused method of controlling pelvic hemorrhage. Am J Obstet Gynecol 1997; 176:938-948

8. Pelage JP, Le Dref O, Jacob D, et al. Selective arterial embolization of the uterine arteries in the management of intractable post-partum hemorrhage. Acta Obstet Gynecol Scand 1999; 78:698-703

9. Ornan D, White R, Pollak J, et al. Pelvic embolization for intractable postpartum hemorrhage: long-term follow-up and implications for fertility. Obstet Gynecol 2003; 102:904-910

10. Wang H, Garmel S. Successful term pregnancy after bilateral uterine artery embolization for postpartum hemorrhage. Obstet Gynecol 2003; 102:603-604

11. McIvor J, Cameron EW. Pregnancy after uterine artery embolization to control hemorrhage from gestational trophoblastic tumor. Br J Radiol 1996; 69:624-629

12. Chow TW, Nwosu EC, Gould DA, Richmond DH. Pregnancy following successful embolisation of a uterine vascular malformation. Br J Obstet Gynaecol 1995; 102:166-168

13. Poppe W, Van Ascher FA, Wilms G, et al. Pregnancy after transcatheter embolization of a uterine arteriovenous malformation. Am J Obstet Gynecol 1987; 156:1179-1180

14. Frates MC, Benson CB, Doubilet PM, et al. Cervical ectopic pregnancy: results of conservative treatment. Radiology 1994; 191:773-775

15. Marston LM, Dotters DJ, Katz VL. Methotrexate and angiographic embolization for conservative treatment of cervical pregnancy. South Med J 1996; 89:246-248

16. Pattinson HA, Dunphy BC, Wood S, et al. Cervical pregnancy following in vitro fertilization: evacuation after uterine artery embolization with subsequent successful intrauterine pregnancy. Aust N Z J Obstet Gynaecol 1994; 34:492-493

17. Vashisht A, Smith JR, Thorpe-Beeston G, et al. Pregnancy subsequent to uterine artery embolization. Fertil Steril 2001; 75:1246-1247

18. Kovacs P, Stangel JJ, Santoro NF, et al. Successful pregnancy after transient ovarian failure following treatment of symptomatic leiomyomata. Fertil Steril 2002; 77:1292-1295

19. Goldberg J, Pereira L, Berghella V. Pregnancy after uterine artery embolization. Obstet Gynecol 2002; 100:869-872

20. D'Angelo A, Amso NN, Wood A. Spontaneous multiple pregnancy after uterine artery embolization for uterine fibroid: a case report. Eur J Obstet Gynecol Reprod Biol 2003; 110:245-246

21. Olive DL, Lindheim SR, Pritts EA. Non-surgical management of leiomyoma: impact on fertility. Curr Opin Obstet Gynecol 2004; 16:239-243

22. Ravina JH, Ciraru-Vigneron N, Aymard A, et al. Pregnancy after embolization of uterine myoma: report of 12 cases. Fertil Steril 2000; 73:1241-1243

23. McLucas B, Goodwin S, Adler L, et al. Pregnancy following fibroid embolization. Int J Gynaecol Obstet 2001; 74:1-7

24. Pron G, Mocarski E, Bennett J, et al. Pregnancy after uterine artery embolization for leiomyomata: the Ontario multicenter trial. Obstet Gynecol 2005; 105:67-76

25. Goldberg J, Pereira L, Berghella V, et al. Pregnancy outcomes after treatment for fibromyomata: uterine artery embolization versus laparoscopic myomectomy. Am J Obstet Gynecol 2004; 191:18-21

26. Walker WJ, McDowell SJ. Pregnancy after uterine artery embolization for leiomyomata: a series of 56 completed pregnancies. Am J Obstet Gynecol 2006; 195:1266-1271

27. Kim MD, Kim NK, Kim HJ, et al. Pregnancy following uterine artery embolization with polyvinyl alcohol particles for patients with uterine fibroid or adenomyosis. Cardiovasc Intervent Radiol 2005; 28:611-615

28. Carpenter TT, Walker WJ. Pregnancy following uterine artery embolisation for symptomatic fibroids: a series of 26 completed pregnancies. BJOG 2005; 112:321-325

29. Holub Z, Mara M, Kuzel D, Jabor A, Maskova J, Eim J. Pregnancy outcomes after uterine artery occlusion: prospective multicentric study. Fertil Steril 2008; 90:1886-1891

30. Spies JB, Ascher SA, Roth AR, et al. Uterine artery embolization for leiomyomata. Obstet Gynecol 2001; 98:29-34

31. Firouznia K, Ghanaati H, Sanaati M, et al. Uterine artery embolization in 101 cases of uterine fibroids: do size, location, and number of fibroids affect therapeutic success and complications. Cardiovasc Intervent Radiol 2008; 31:521-526

32. Andrews RT, Brown PH. Uterine arterial embolization: factors influencing patient radiation exposure. Radiology 2000; 217:713-722

33. Descargues G, Mauger Tinlot F, Douvrin F, Clavier E, Lemoine JP, Marpeau L. Menses, fertility and pregnancy after arterial embolization for the 


\section{Pregnancy After Uterine Artery Embolization}

control of postpartum haemorrhage. Hum Reprod 2004; 19:339-343

34. Spies JB, Roth AR, Gonsalves SM, et al. Ovarian function after uterine artery embolization for leiomyomata: assessment with use of serum follicle stimulating hormone assay. J Vasc Interv Radiol 2001; 12:437-442

35. Walker WJ, Pelage JP. Uterine artery embolisation for symptomatic fibroids: clinical results in 400 women with imaging follow-up. BJOG 2002; 109:1263-1272

36. Pron G, Bennett J, Common A, et al. The Ontario Uterine Fibroid Embolization Trial. Part 2. Uterine fibroid reduction and symptom relief after uterine artery embolization for fibroids. Fertil Steril 2003; 79:120-127

37. Chrisman HB, Saker MB, Ryu RK, et al. The impact of uterine fibroid embolization on resumption of menses and ovarian function. J Vasc Interv Radiol 2000; 11:699-703

38. Payne JF, Robboy SJ, Haney AF. Embolic microspheres within ovarian arterial vasculature after uterine artery embolization. Obstet Gynecol 2002; 100:883-886

39. Tulandi T, Sammour A, Valenti D, et al. Ovarian reserve after uterine artery embolization for leiomyomata. Fertil Steril 2002; 78:197-198

40. Tropeano G, Di Stasi C, Litwicka K, Romano D,
Draisci G, Mancuso S. Uterine artery embolization does not have adverse effects on ovarian reserve in regularly cycling women younger than 40 years. Fertil Steril 2004; 81:1055-1061

41. deSouza NM, Williams AD. Uterine arterial embolization for leiomyomas: perfusion and volume changes at MR imaging and relation to clinical outcome. Radiology 2002; 222:367-374

42. Pelage JP, Guaou NG, Jha RC, Ascher SM, Spies JB. Uterine fibroid tumors: long-term MR imaging outcome after embolization. Radiology 2004; 230:803-809

43. Toner JP, Flood JT. Fertility after the age of forty. Obstet Gynecol Clin North Am 1993; 20:261-272

\section{F OR Y O UR INFORMATION}

The reader's attention is directed to the commentary on this article, which appears on the following pages. 